\title{
Corela
}

Cognition, représentation, langage

HS-18 | 2015

La reformulation : usages et contextes

\section{La reformulation : de la construction du sens à la construction des apprentissages en langue et sur la langue}

Claire Martinot

\section{OpenEdition}

Journals

Édition électronique

URL : https://journals.openedition.org/corela/4034

DOI : $10.4000 /$ corela.4034

ISSN : 1638-573X

Éditeur

Cercle linguistique du Centre et de l'Ouest - CerLICO

Édition imprimée

Date de publication : 15 novembre 2015

ISSN : 1638-5748

Référence électronique

Claire Martinot, « La reformulation : de la construction du sens à la construction des apprentissages en langue et sur la langue », Corela [En ligne], HS-18 | 2015, mis en ligne le 15 novembre 2015, consulté le 21 septembre 2021. URL : http://journals.openedition.org/corela/4034 ; DOI : https://doi.org/ 10.4000/corela.4034

Ce document a été généré automatiquement le 21 septembre 2021.

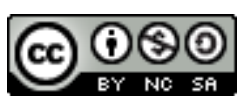

Corela - cognition, représentation, langage est mis à disposition selon les termes de la licence Creative Commons Attribution - Pas d'Utilisation Commerciale - Partage dans les Mêmes Conditions 4.0 International. 


\title{
La reformulation : de la construction du sens à la construction des apprentissages en langue et sur la langue
}

\author{
Claire Martinot
}

\section{Cadre d'analyse de la reformulation}

1 Le concept de reformulation est apparu, d'abord, dans le cadre de l'étude des marqueurs de reformulation (Gülich et Kotschi, 1983) avec un sens assez proche de celui de paraphrase mais surtout avec une application réservée à la pratique orale de la langue et plus particulièrement au genre conversationnel. En effet, depuis le début des années 1980, un nouveau type d'analyse des énoncés paraphrastiques, en langue parlée, a fait son apparition avec les travaux de Ströbl (1977), Warmhoff (1981), Wenzel (1981), Gülich et Kotschi (1983) en Allemagne et de Martin (1976) ou Fuchs $(1978,1980,1982)$ en France. Ces travaux, d'obédience plutôt sémantique, décrivent différents types de paraphrases dans la langue parlée et on peut sans doute voir dans cet objet de recherche une certaine filiation, même si la perspective est très différente, avec les travaux princeps de Harris $(1970,1976)$ qui fournissent une explication au fonctionnement général des langues à partir des relations transformationnelles que l'on peut établir entre les phrases d'une langue: Harris (1969) considère que les langues sont constituées de deux classes distinctes d'équivalence, le Report qui correspond à la réunion d'un énoncé quelconque et d'un énoncé descripteur (sémantiquement équivalent, non utilisé dans la pratique des locuteurs du fait de son caractère saturé par la redondance), et la Paraphrase, qui correspond à la réunion de deux énoncés sémantiquement équivalents de même statut ${ }^{1}$.

Mais l'observation attentive de grands corpus en langue parlée ${ }^{2}$ montre que les reprises sont aussi bien des paraphrases, plus ou moins approximatives (1-2) ci-dessous, que des énoncés exprimant un sens différent, au sens d'information différente (3-5)3: 
(1) [Enoncé 1] [J'ai toujours aimé mieux les maths que le que le reste] + j'étais quand même bonne en français mais [énoncé 2] [c'est les maths qui qui me plaisaient le plus]

(2) [Enoncé 1] [Il y avait le buraliste + bien sûr qui faisait partie du + du milieu aussi puisqu'on allait lui acheter chaque semaine le jeudi] à l'époque hein + puisque le mercredi n'existait pas c'était un jour de travail pour les écoliers + donc euh [énoncé 2] [le buraliste chez qui on allait acheter les journaux ou le tabac pour les parents le le jeudi] voilà

(3) [Enoncé 1] [ils allaient à Mouthe] [Enoncé 2] [ils allaient à Morez] [Enoncé 3] [ils allaient à Morbier] [Enoncé 4] + [ils allaient chercher la farine pour faire le pain tout]

(4) [Enoncé 1] [Ils ne mangeaient que les feuilles vertes hein] [Enoncé 2] [ils ne mangeaient pas le cœur ]

(5) [Enoncé 1] [On faisait du feu] [Enoncé 2] [on faisait griller nos pommes de terre]

3 La diversité des modes de reprise dans la langue parlée avait déjà conduit Gülich et Kotschi (1987) à utiliser le terme de reformulation plutôt que celui de paraphrase, sans aller jusqu'à affirmer qu'un énoncé reformulé pouvait exprimer un sens différent relativement à un énoncé antérieur reconnu comme étant la source. Pour pouvoir rendre compte de tous les types de reformulation, y compris ceux qui modifient le sens de l'énoncé source, comme en (3-5) ci-dessus, une définition opératoire de ce concept est donc nécessaire (Martinot \& Romero, 2009: 9-12).

4 Nous proposerons, ou nous rappellerons, cette définition (Martinot, 1994) et la justifierons. Nous verrons ensuite que cette définition permet de rendre compte des différentes postures reformulatoires que l'enfant adopte au cours de l'acquisition de sa langue maternelle. Enfin, si l'on admet que l'enfant acquiert sa langue maternelle en transformant les énoncés adultes qui lui sont accessibles et que cette posture reformulatoire est naturelle - en-effet les reformulations produites par les locuteurs sont alimentées par un mode d'organisation paraphrastique déjà présent dans la langue - alors on a intérêt à utiliser et à systématiser cette posture spontanée dans le cadre pédagogique avec pour objectif de faire prendre conscience aux élèves des contraintes de fonctionnement de la langue qui opèrent aux niveaux lexical, morphologique, syntaxique, sémantique et pragmatique.

5 Les recherches que nous menons sur la reformulation, chez les adultes (Martinot, 2004, 2005b, sous presse) et chez les enfants (ci-dessous) tentent de répondre aux questions soulevées par Gülich et Kotschi (ibid.) et ne s'inscrivent pas nécessairement dans le contexte des interactions en milieu scolaire, comme c'est le cas des articles de cette livraison, qui recherchent quelles fonctions didactiques remplissent les reformulations de l'enseignant, ou bien quelles trajectoires langagières suivent les élèves pour construire leurs connaissances à partir des échanges oraux dans la classe. Dans le domaine didactique que nous aborderons cependant dans la dernière partie de cet article, nous proposons d'envisager la reformulation en tant qu'activité à part entière d'apprentissage de la langue, de façon à amener les élèves à expérimenter le fonctionnement de la langue.

\section{Définition technique de la reformulation}

6 Nous nous appuierons sur la définition de Martinot (1994) que nous rappelons: "Tout processus de reprise d'un énoncé antérieur qui maintient, dans l'énoncé reformulé, une partie invariante à laquelle s'articule le reste de l'énoncé, partie variante par rapport à l'énoncé source, est une reformulation ». Cette définition permet de rendre compte d'une 
part des reformulations paraphrastiques, non paraphrastiques et répétitives ${ }^{4}$. D'autre part, de situer le niveau linguistique de l'invariant, (niveau lexical, syntaxique ou sémantique) concerné par la reformulation entre l'énoncé source et l'énoncé reformulé, de même que le niveau linguistique de la partie modifiée introduite dans l'énoncé reformulé. Ainsi par exemple, un énoncé reformulé peut garder le même lexique et la même construction mais pas le même sens (6) :

(6) (ES) le garçon regarde la fille > (ER) la fille regarde le garçon

7 Un énoncé reformulé peut garder le même lexique et le même sens, mais pas la même construction: cas des transformations, cf. (7), ou pas la même structure: cas des restructurations, $\mathrm{cf}$. (8) :

(7) (ES) cette affaire concerne mon voisin > (ER) mon voisin est concerné par cette affaire

(8) (ES) Luc a chargé les caisses dans le camion > (ER) Luc a chargé le camion de caisses

8 Un énoncé reformulé peut garder le même sens mais ni le même lexique, ni la même construction : cas des paraphrases sémantiques, cf. (9):

(9) (ES) le juge interroge le suspect > (ER) le magistrat pose des questions au prévenu

La définition technique ci-dessus est fondée sur un principe unique qui rassemble tous les types de reformulation ${ }^{5}$ et s'appuie sur une force créative et une force d'invariance à l'œuvre simultanément entre un énoncé source et un énoncé reformulé. La force créative transforme l'énoncé source (en modifiant le lexique et/ou la construction et/ou le sens) tandis que la force d'invariance met en relation - par la manifestation ou la reconnaissance de ce qui ne change pas - les deux énoncés.

10 Cette définition permet aussi de classer les types de reformulation (Martinot et al, 2008, Martinot, 2012, Martinot, sous presse).

11 Elle permet ensuite de montrer comment les locuteurs construisent préférentiellement le sens de leurs énoncés relativement à des énoncés antérieurs. Si la reprise des mots de l'autre, des énoncés de l'autre est un phénomène banal et inéluctable, la répétition stricte de séquences prédicatives est, sauf cas très particuliers, impropre à construire un sens quelconque. On ne peut faire sens qu'en introduisant un différentiel par rapport à un énoncé source (force créative du principe reformulatoire) tout en établissant un minimum de relation avec un énoncé antérieur - on ne fait pas sens à partir de rien (force d'invariance du principe reformulatoire). Les reformulations paraphrastiques et non paraphrastiques sont donc celles qui construisent a priori le sens, qu'il s'agisse d'apporter un nouveau point de vue, un éclairage différent pour une même information (reformulations paraphrastiques) ou de donner une autre information avec la même construction (reformulations non paraphrastiques).

\section{Rôle de la reformulation dans l'acquisition de la langue}

12 S'il y a une totale unanimité parmi les spécialistes de l'acquisition de la langue maternelle pour dire que l'enfant n'apprend à parler que s'il est entouré de personnes qui lui parlent, le mécanisme qui permet à l'enfant de passer des énoncés qui lui sont adressés aux énoncés qu'il produit ne fait pas l'objet d'un consensus. Sans entrer dans les nombreux et 
longs débats, par ailleurs nécessaires, sur le processus acquisitionnel, nous proposons une explication transformiste, qui renouvelle les paradigmes générativiste, cognitiviste et fonctionnaliste. Elle s'appuie sur l'attitude, la posture reformulatoire des jeunes locuteurs telle que nous l'avons observée et qui nous a conduit à la définition de la reformulation (cf. partie 1, ci-dessus). L'idée directrice est que l'enfant transforme de façon non aléatoire les énoncés qui lui sont adressés ou les énoncés construits à plusieurs ${ }^{6}$. Cette hypothèse explicative donne un rôle négligeable à la répétition et un rôle déterminant à la transformation. Elle s'appuie aussi sur la théorie harrissienne (Harris, 1976) selon laquelle une langue est constituée de discours et non de mots, et que ces discours (phrases plus ou moins bien formées grammaticalement) sont en relation transformationnelle les uns avec les autres. La conséquence de cette vision du fonctionnement de la langue (adressée à l'enfant) est que premièrement l'enfant entend des prédications (Ibrahim, 2009:12) ${ }^{7}$, qui lui disent souvent la même chose mais sous des formes un peu différentes, que deuxièmement il produit lui-aussi, et dès ses premières productions linguistiques, une fois la phase du babillage révolue, des prédications et non des mots. Cette affirmation peut sembler contre-intuitive puisque chacun peut constater que l'enfant produit pendant plusieurs mois des énoncés réduits à un mot (conventionnel ou non) puis des énoncés de deux mots puis des énoncés de trois mots (cette vision mécaniciste du développement du langage n'explique pas l'émergence de la prédication). Et c'est seulement à partir de ce nombre moyen (trois) de mots par énoncé que les chercheurs commencent à parler de "phrases " (De Boysson-Bardies, 1996 : 249). Nous pensons que les premières productions linguistiques sont des prédications, même si elles sont incomplètes, dans la mesure où l'enfant veut faire sens et où le seul mot de son énoncé est la trace d'une prédication qui était complète lorsque l'enfant l'a entendue.

La première période acquisitionnelle, dans notre hypothèse, correspond aux deux-trois années pendant lesquelles l'enfant apprend à construire des prédications de plus en plus complètes, grosso modo de 1 an et quelques mois à 4 ans, au plus tard:

« coucou » > "fais/t coucou » > "fais/t coucou à Papa » > "Arthur, fais/t coucou à Papa!» 《 Arthur fait coucou à Papa».

Le mécanisme qui permet à l'enfant de produire ces prédications successives vient des reformulations simplificatrices qu'il a faites après avoir entendu sa mère lui dire : « Fais coucou à Papa!» ou dans un commentaire: "Arthur fait coucou à Papa». On doit évidemment se demander comment l'enfant reconnaît dans l'énoncé adulte qui lui est adressé quel est le mot prédicatif. Nous n'avons pas de réponse à cette question particulièrement importante, et restons, comme Chomsky, émerveillée, devant cette faculté humaine, qu'a le petit enfant de reconnaître dans une suite de phonèmes (correspondant au minimum à une prédication) qui lui est adressée quel est le segment de cette suite qui porte la charge prédicative de la phrase (ici, coucou) ${ }^{8}$.

Mais une fois que l'enfant sait construire des prédications simples complètes (aux alentours de 3 ans), une autre question tout aussi énigmatique doit être résolue : comment l'enfant s'y prend-il pour continuer à simplifier les prédications adultes qu'il réutilise? Une simple comparaison quantitative d'énoncés adultes et enfantins (enfants de 6-7 ans) produits au cours de la même tâche (récits de vie) suffit à constater que les énoncés enfantins sont beaucoup plus simples que les énoncés adultes. Cela ne signifie pas que les enfants n'emploient pas de subordonnées - ils emploient certains types de subordonnées - mais que leurs prédications ne renferment qu'un prédicat et ne comportent que les éléments indispensables à la grammaticalité des énoncés (Martinot, 
2005a). Les enfants utilisent donc la reformulation, au-moins jusqu'à l'âge de 6-7 ans, pour simplifier la langue de leur environnement de façon à pouvoir se l'approprier. Mais à 6-7 ans, l'acquisition de la langue est encore loin de son terme. Le processus acquisitionnel se poursuit ensuite du fait de l'application de nouvelles procédures de reformulation qui servent à complexifier les énoncés déjà maîtrisés par les enfants ou à reformuler les énoncés complexes des adultes. Cette hypothèse acquisitionnelle a été testée dans plusieurs langues maternelles ${ }^{9}$, le français, l'italien, le croate, l'allemand, le polonais, le roumain et l'arabe yéménite. Nous présentons ci-dessous les principaux résultats de ce projet, concernant le français.

\section{Nécessité d'une procédure expérimentale pour vérifier l'hypothèse d'une acquisition par reformulation : Projet international Acquisition et Reformulation}

17 Le repérage de la paire [énoncé source-énoncé reformulé] à l'oral n'est pas chose aisée. En dehors des situations privilégiées d'un dialogue entre un petit enfant et sa mère qui révèlent comment d'une part les reformulations de la mère, d'autre part les reformulations de l'enfant (Leroy-Collombel, 2009) contribuent à la construction du sens, le repérage, par le chercheur, des paires [énoncé source-énoncé reformulé] devient vite impossible. Une situation expérimentale suffisamment proche de la situation linguistique que vit l'enfant doit donc être envisagée. Les hypothèses de recherche testées dans ce projet sont les suivantes:

18 1. Les enfants reformulent les énoncés sources qui leur servent de modèles de façons très diverses. Cette diversité est en grande partie liée à l'âge des enfants (4, 6, 8 et 10 ans) et donc à leurs compétences transformistes. On devrait constater une évolution dans les procédures de reformulation en fonction de l'âge. On vérifiera que cette évolution va grosso modo des reformulations répétitives aux reformulations paraphrastiques.

19 2. On devrait également constater une évolution dans les types de reformulation paraphrastique: certains étant plus précoces que d'autres (les reformulations définitoires) et donc vraisemblablement plus faciles à mettre en œuvre (Martinot, 2009, Gerolimich, Martinot, De Gioia, 2009, Martinot \& Gerolimich, 2012)

3. On devrait trouver des postures de reformulation comparables d'une langue à l'autre chez les enfants de même âge (Martinot et al, 2009) mais également des différences provenant des zones de complexité qui ne sont pas les mêmes d'une langue à l'autre (en français, par ex. l'énoncé elle tenait une petite fille par la main a été restructuré de multiples façons dès 4 ans, tandis qu'en italien, l'énoncé équivalent teneva per mano una bambina n'acceptant pas les mêmes transformations, les enfants italophones ont eu des difficultés à le reformuler quand ils avaient oublié la forme exacte de l'énoncé source). De la même façon, dans une même langue, les zones de complexité seront traitées de façons différentes et réserveront des difficultés aux jeunes enfants et peut-être aussi aux enfants qui, à âge égal, ne bénéficiant pas d'une stimulation linguistique suffisante, reformulent peu les énoncés sources complexes (Martinot, 2013).

21 Ces résultats sont susceptibles de fournir des outils permettant de distinguer différents stades acquisitionnels chez les enfants, au moins jusqu'à l'âge de 10 ans. Ces outils 
peuvent donc être utilisés et systématisés dans l'enseignement du français à l'école élémentaire comme nous le verrons à travers quelques exemples à la fin de cet article.

\subsection{Protocole expérimental utilisé dans le projet Acquisition et Reformulation}

22 A la suite de la lecture ${ }^{10}$ d'une histoire (cf. annexe), l'enfant est invité à la restituer immédiatement après avec «ses mots à lui et en essayant de ne rien oublier ». L'intérêt de ce type de tâche est double: 1 . le chercheur contrôle le texte source, il peut donc observer, dans les transcriptions, comment l'enfant a transformé chaque prédication de ce texte et comparer un grand nombre d'enfants dans les mêmes conditions; 2 . le différentiel introduit par l'enfant lors de sa reformulation de l'histoire ne devrait pas être différent de celui qu'il introduit lorsqu'il reformule un énoncé adulte complexe qu'il s'approprie par la reformulation parce que ce différentiel correspond à ce que l'enfant sait faire avec la langue d'une façon générale.

L'histoire racontée a été rédigée par l'auteur de ces lignes. Pour s'assurer que les enfants de 4 ans comprenaient l'histoire, l'auteur a demandé à un enseignant de moyenne section d'une école maternelle de la banlieue parisienne située en zone sensible de lire l'histoire à ses élèves et de les faire parler de cette histoire ensuite. Le test a été concluant. La même histoire a été lue à des enfants de 10 ans qui l'ont écoutée avec grand intérêt. En-effet, le choix d'un texte source adapté à des enfants de 4 à 10 ans doit prendre en compte à la fois la capacité de compréhension des plus petits et correspondre encore aux centres d'intérêt des plus grands. Par ailleurs, l'histoire doit pouvoir concerner des enfants de tous pays puisque cette expérimentation a pour vocation de comparer l'acquisition de différentes langues maternelles. C'est pourquoi l'auteur a choisi de situer l'histoire dans un contexte scolaire, familier aux enfants européens au-moins, avec comme seul représentant du monde adulte, l'enseignante ${ }^{11}$. Enfin, l'histoire comporte un grand nombre de phénomènes complexes ${ }^{12}$ (compléments facultatifs antéposés, relatives, gérondif, noms complexes de type $\mathrm{N}$ de $\mathrm{N}$, verbes sémantiquement complexes, métaphores...).

\subsection{Choix des locuteurs et conditions d'enregistrement}

24 Le corpus analysé rassemble 4 tranches d'âge : 4, 6, 8 et 10 ans avec une fourchette de 3 mois pour chaque tranche d'âge (ex. 4;0 à 4;3). Dans chaque tranche d'âge, 15 restitutions sont analysées. Les enfants sont francophones et ne présentent pas de caractéristiques particulières, ils sont scolarisés soit en maternelle, soit en élémentaire et les enregistrements ont généralement lieu dans les écoles. Il est hautement souhaitable que l'enfant connaisse bien (et apprécie) l'adulte qui lui lit l'histoire, en particulier avec les 4 ans. Le texte lu ne comporte pas d'images, la lecture doit être faite de façon théâtralisée. Les enfants sont volontaires pour écouter et raconter. L'adulte est seul avec l'enfant, dans un endroit calme. Lors de la restitution faite par l'enfant, l'adulte ne doit pas intervenir, sauf pour encourager ou féliciter l'enfant, de façon à ce que celui-ci comprenne bien qu'il doit raconter l'histoire tout seul, sans l'aide de l'adulte. L'immense majorité des enfants s'est prêtée de très bonne grâce à ce petit « jeu ", à l'exception des 4 ans de certains pays dans lesquels la scolarisation à cet âge n'est pas la règle. 


\subsection{Mode d'analyse}

Une fois les transcriptions faites, il s'agit donc d'analyser comment chaque enfant a reformulé chaque prédication du texte source. En effet, pour pouvoir évaluer si l'énoncé reformulé est une paraphrase, une répétition ou s'il change le sens de l'énoncé source, il faut segmenter le texte source (désormais TS) de même que le texte reformulé (désormais TR) en prédications, simples ou complexes. Cette condition syntaxique est indispensable pour procéder à l'analyse des reformulations. Exemples extraits des reformulations de la séquence 1 (ou énoncé source) (cf. annexe) :

Enoncé source (ES) : Ce matin-là, la maîtresse est arrivée dans la cour de l'école plus tard que d'habitude. Elle tenait par la main une petite fille que personne n'avait encore jamais vue.

Prédication 1 : (ce matin-là) la maîtresse est arrivée dans la cour de l'école

Prédication 2 : (ce matin-là) la maîtresse est arrivée plus tard que d'habitude

Prédication 3 : elle tenait par la main une petite fille

Prédication 4 : que personne n'avait encore jamais vue.

Remarque : La première phrase (segment allant d'un point à un point) de la séquence 1 de l'histoire est une prédication complexe, constituée de deux prédications élémentaires (prédications 1 et 2). La deuxième phrase de l'histoire est aussi une prédication complexe constituée d'une principale et d'une relative (prédications 3 et 4).

Exemples de reformulations :

Adrien (4 ans) : ER. La maîtresse rentrait plus tard que d'habitude + elle tenait une petite fille que tout le monde n'avait jamais vue ${ }^{13}$

Prédication $2:$ La maîtresse rentrait plus tard que d'habitude

Prédication 3 : elle tenait une petite fille

Prédication 4 : que tout le monde n'avait jamais vue

Une fois ce séquençage fait, chaque prédication reformulée est comparée à la prédication source. Il est recommandé de rassembler, à la suite d'un ES, tous les énoncés reformulés des enfants de même âge de façon à repérer plus facilement les constantes. Exemple d'analyse comparative concernant uniquement la prédication 2 de l'ES :

ES : la maîtresse est arrivée plus tard que d'habitude

ER (Adrien, 4) : La maîtresse rentrait plus tard que d'habitude

ER (Pauline, 4) : la maîtresse vena très tard

ER (Antoine, 4) : Au fait il y a une maîtresse qui est venue (...) très tard

ER (Marion, 4) : Il y avait la maîtresse qui rentrait plus tard que d'habitude

On évalue dans un premier temps si la procédure de reformulation attestée est une répétition (stricte ou approximative), si l'enfant a modifié le sens ou si l'enfant a fourni une équivalence sémantique. Dans un deuxième temps, on caractérise le type de paraphrase, nous en verrons des exemples plus loin.

La principale difficulté pour le chercheur est de repérer le mouvement reformulatoire majeur qui affecte globalement la prédication. Une partie de la prédication source peut en effet être répétée dans l'ER, en présence d'un verbe qui modifie le sens de la prédication, ou encore on peut avoir globalement la même information même si l'un des constituants a été sémantiquement modifié : par ex. un enfant a reformulé une petite fille par une jeune fille.

31 Dans les exemples de reformulation ci-dessus, Adrien a répété le sujet, le complément temporel, mais a modifié le verbe. Dans le contexte de l'histoire, ce verbe nouveau ne 
modifie pas le sens de la prédication (l'information n'est pas contradictoire avec celle de l'ES), on dira donc que l'enfant a répété la prédication 2 en changeant néanmoins le lexique verbal (répétition approximative).

Pauline modifie également le verbe et change la nature de l'information temporelle, ce qui entraîne un changement de sens pour l'ensemble de la prédication reformulée.

Antoine modifie la construction, modifie le verbe ainsi que la nature de l'information temporelle, c'est donc une reformulation par changement de sens qui sera proposée.

Enfin, Marion modifie la construction de la prédication source, le verbe et répète le complément temporel. Au niveau de l'information transmise, Marion fournit une reformulation proche de celle d'Adrien, mais elle s'en distingue par le changement de construction. On a donc dans ce cas une équivalence sémantique.

Voyons maintenant des exemples de reformulation qui mettent en œuvre les différents types de paraphrases : paraphrase formelle (sous-type : transformation, cf. Alexandrine \& sous-type : restructuration, cf. Léo), paraphrase sémantique, cf. Paul:

Séquence 11 : Tout à coup, le tronc s'ouvrit et les enfants furent éblouis par la lumière qui inondait l'intérieur de l'arbre. Ils firent quelques pas et l'arbre se referma derrière eux.

Prédication $1:$ (Tout à coup) le tronc s'ouvrit

Prédication 2 : (et) les enfants furent éblouis par la lumière

Prédication $3:$ qui inondait l'intérieur de l'arbre.

Prédication 4 : Ils firent quelques pas

Prédication $5:$ (et) l'arbre se referma derrière eux

ES: les enfants furent éblouis par la lumière qui inondait l'intérieur de l'arbre (prédications 2 et 3 )

ER (Alexandrine, 10) : une grande lumière l'éblouit (prédication 2)

ER (Léo, 10) : à l'intérieur c'est inondé de lumière (prédication 3)

ER (Paul, 10) : les enfants éblouis par la lumière qui sortait du tronc (prédications 2

et 3)

Alexandrine transforme la prédication 2 en changeant la voix : passif > actif. Il s'agit donc d'une transformation, c'est-à-dire d'une paraphrase formelle, de cette prédication, les autres changements sont négligés.

Léo restructure la prédication $3 \mathrm{du}$ fait que l'intérieur n'a plus la même position syntaxique que dans le TS. Par ailleurs, il transforme la voix active de la prédication source (la lumière inondait ...) en passif et la construction personnelle de la relative source devient impersonnelle. Ces modifications difficiles à réaliser correspondent à une restructuration du complément direct l'intérieur en complément locatif, reformulation qui englobe d'une part la transformation actif-passif (inondait > est inondé) et d'autre part la transformation impersonnelle.

Enfin, Paul répète quasiment la prédication 2 et produit une paraphrase sémantique très approximative de la prédication 3 (on pourrait bien entendu considérer qu'il y a changement de sens dans l'absolu : inonder un endroit \# sortir de cet endroit, cependant nous préférons considérer que la même information est donnée sous une autre forme (paraphrase sémantique approximative). La lumière provient bien du tronc, d'autre part le trop plein évoqué par le verbe inonder implique bien que la lumière puisse en sortir.

Le troisième type de paraphrase, la paraphrase définitoire, est attesté, par exemple, dans les reformulations de la séquence 2 :

ES : (...) Julie sera ta voisine (...)

ER (Chloé, 6) : la maîtresse elle lui dit de se mettre à côté de Tom 
considère en effet que Julie sera la voisine de Tom est défini par Julie se met à côté de Tom dans la mesure où l'enfant qui s'assoit à côté d'un autre enfant en classe devient son voisin/sa voisine ${ }^{14}$ (Ibrahim \& Martinot, 2004 ; Gerolimich, Martinot, De Gioia, 2009).

41 Ou dans celles de la séquence 4 :

ES : (...) lui a tendu la boîte qu'il avait fabriquée pour elle, la veille.

ER (Gaël, 6) : (le lendemain matin dans la cour de récréation) Tom *tenda le bras et euh Julie *prena la boîte

L'analyse définitoire (Gerolimich et al, 2009) du prédicat tendre un objet correspond à tendre le bras vers quelqu'un de façon à ce que cette personne prenne l'objet. Ce type de reformulation définitoire manifeste de façon très convaincante comment l'enfant construit le sens en discours, en décomposant le sens qui est condensé dans tendre un objet à quelqu'un. 1 : l'équivalence entre petite fille que personne n'avait encore jamais vue et petite fille qui faisait sa rentrée à l'école n'est valable qu'en discours, dans le contexte de l'histoire mais surtout correspond à l'explication que donne l'enfant de l'information source (personne n'avait jamais vu cette petite fille parce qu'elle faisait sa rentrée à l'école (pour la première fois)) :

ES : Elle tenait par la main une petite fille que personne n'avait encore jamais vue ER (Maxime, 10) Il était une fois une petite fille qui faisait sa rentrée à l'école

\section{Résultats}

Le mode d'analyse illustré ci-dessus a été appliqué aux 60 enfants francophones de 4, 6, 8 et 10 ans. Les principaux résultats peuvent être énoncés comme suit ${ }^{16}$ :

\subsection{Les procédures principales de reformulation diffèrent tendanciellement selon les âges, ce qui permet de classer ces procédures de la plus simple à la plus difficile à mettre en œuvre}

A 6 et 8 ans, les reformulations qui modifient le sens sont relativement plus nombreuses que chez les 4 et les 10 ans, c'est à 6 et 8 ans que les enfants semblent être le plus inventifs et explorent au maximum leur langue, ce qui a souvent pour conséquence qu'un changement de forme entraîne aussi un changement de sens ; A partir de 8 ans, les reformulations paraphrastiques sont la règle ;

A 10 ans, il n'y a quasiment plus ni répétition, ni changement de sens mais uniquement des reformulations paraphrastiques : définitoire, sémantique, formelle et explicative. 


\subsection{Les reformulations paraphrastiques diffèrent selon l'âge, ce qui permet également de classer les paraphrases produites dans un ordre croissant de difficulté.} imprévisible.
Même si les reformulations paraphrastiques ne sont pas majoritaires à 4 et 6 ans, elles sont suffisamment nombreuses pour que l'on puisse constater quel type de reformulation paraphrastique est employé tendanciellement à cet âge :

Dès 4 ans sont attestées les paraphrases définitoires ;

A 6 et 8 ans sont attestées, en plus, les paraphrases sémantiques ;

A 8 ans apparaissent, en plus, les paraphrases formelles (transformations) ;

A 10 ans apparaissent les restructurations (second type de paraphrases formelles) tandis que les autres types de paraphrases perdurent.

Les paraphrases explicatives sont tendanciellement tardives (nombreuses à 10 ans).

Les reformulations définitoires permettent, selon nous, de produire ultérieurement les autres types de paraphrases, comme si le prototype des paraphrases était la paraphrase définitoire (proche du Report de Harris, 1969, et des Matrices Analytiques Définitoires de Ibrahim, 2001, 2015). Ces reformulations peuvent prendre des formes extrêmement variées (cf. Gerolimich, Martinot, De Gioia, 2009), elles ne sont pas du tout contraintes formellement et présentent, de ce point de vue, peu de difficultés (majeures). Il est tout de même remarquable, qu'avant tout entraînement scolaire, de nombreux enfants de 4 ans soient capables de fournir à partir d'un énoncé source un autre énoncé qui contienne une sorte de définition naturelle (Martin, 1990) de l'un des mots importants de l'énoncé source, cf. Alexandre ci-dessous : Il lui était interdit d'aller > c'est interdiction d'y aller :

ES. (Séq. 7) Tom avait un peu peur parce qu'il lui était interdit d'aller dans la forêt, surtout la nuit.

ER. (Alexandre, 4) après Tom il croyait que ce n'était pas un une bonne idée parce que c'est interdiction d'y aller euh ++ après après après il avait un peu peur.

Les paraphrases sémantiques n'obéissent qu'à la contrainte de l'équivalence sémantique, ce qui explique qu'elles soient d'un usage relativement précoce. Elles ne présentent pas de caractère définitoire mais en revanche révèlent dans quelle mesure les enfants peuvent faire varier le lexique (chuchoter quelque chose > souffler quelque chose). On remarquera dans cet exemple que les deux verbes ne sont pas tout à fait équivalents même s'ils partagent une partie de leur définition analytique : dire quelque chose à voix basse. L'équivalence sémantique, dans une tâche de restitution d'histoire, est le plus souvent approximative.

A l'inverse des paraphrases définitoires et sémantiques, la difficulté pour n'importe quel locuteur de produire une paraphrase formelle (transformation), vient du fait que le même lexique est repris dans une construction différente (passif vs actif, permutation d'un constituant, nominalisation d'un verbe...) mais ce changement de construction a un caractère relativement régulier et peu dépendant du lexique.

Dans les restructurations (second type de paraphrases formelles), le lexique est également repris à la prédication source mais la structure argumentale du verbe, le plus souvent, est modifiée ${ }^{17}$. Cette modification est dépendante du lexique et donc 
Pour les restructurations, la contrainte formelle est maximale puisque la structure doit être compatible avec le lexique et préserver la même information.

Enfin, les paraphrases explicatives ne présentent pas de contraintes formelles, en revanche, elles introduisent un nouveau point de vue, une cause, une conséquence ou une justification. Elles nécessitent une grande maitrise textuelle, et/ou une connaissance du monde et des contraintes pragmatiques que ne peuvent encore avoir les jeunes enfants. Ainsi par exemple, on ne trouve que chez les enfants de 10 ans, des cas de paraphrases explicatives de que personne n'avait encore jamais vue:

ES : (séq. 1) Ce matin-là, la maîtresse est arrivée dans la cour de l'école plus tard que d'habitude. Elle tenait par la main une petite fille que personne n'avait encore jamais vue

ER (Maurine, 10) : C'est l'histoire d'une petite fille qui vient pour la première fois dans une école

ER (Théo, 10) : Alors c'est l'histoire de Tom et de Julie et c'est Tom et Julie donc c'est Julie qui entre dans une nouvelle école

Tableau 1. Procédures de reformulation classées de la moins complexe à la plus complexe

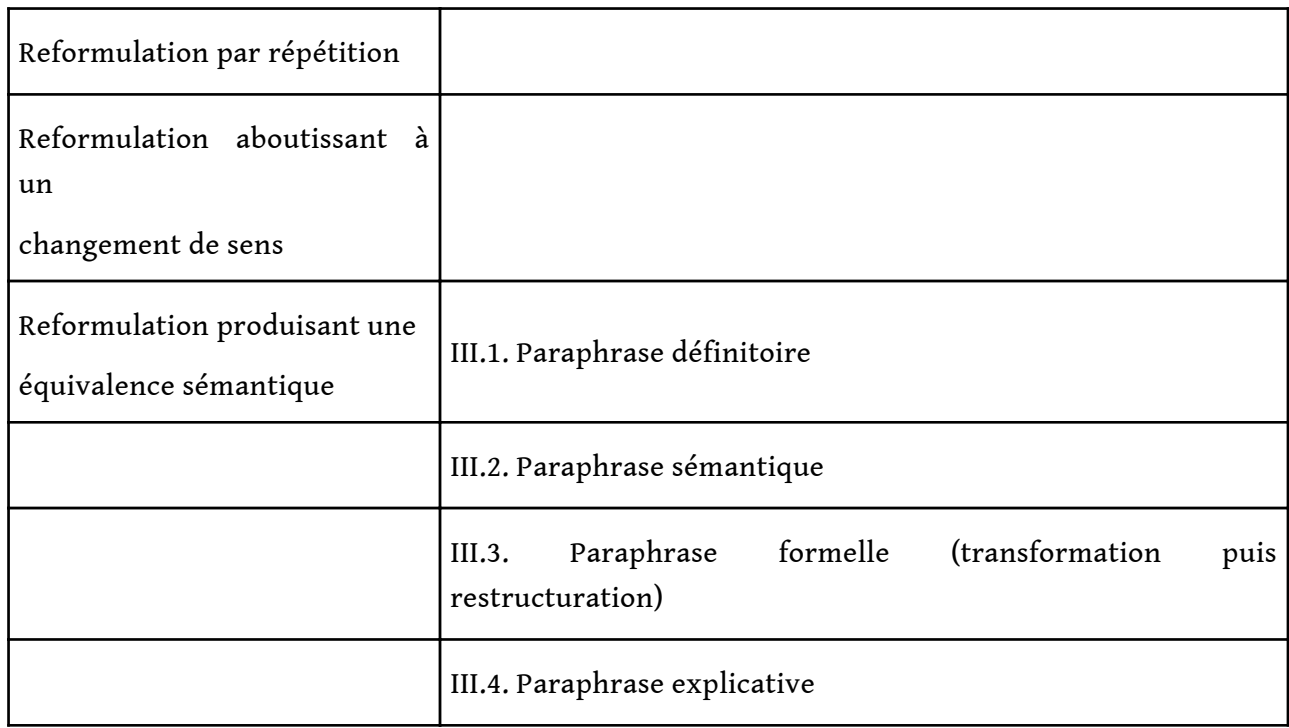

\subsection{De la simplification vers la complexification}

61 Nous avons dit plus haut que les procédures de reformulation permettaient aux enfants de simplifier, dans un premier temps, les énoncés complexes qu'ils reformulent, et dans un deuxième temps, de complexifier les énoncés simples dont ils disposent déjà, ou encore de reformuler avec le même degré de complexité les énoncés complexes qu'ils entendent.

Dans l'analyse que nous faisons des procédures de reformulation, nous distinguons la complexité inscrite dans les énoncés, de la complexité de la procédure mise en œuvre.

Concernant la complexité inscrite dans la langue, elle peut se manifester au niveau de la macro-syntaxe sous différentes formes : par exemple lorsqu'un énoncé est constitué de plusieurs prédications qui s'enchaînent, ou qui s'enchaînent selon des modalités à chaque fois différentes: une relative puis une causative, puis une concessive, puis un 
commentaire ... Les relations anaphoriques et les contraintes de cohérence constituent un aspect supplémentaire de la complexité macro-syntaxique.

$\mathrm{Au}$ niveau micro-syntaxique, la complexité résulte, dans l'approche harrissienne, du nombre de prédicats contenus dans la phrase (unité prédicative simple ou complexe). Autrement dit, une phrase à un prédicat est simple (il y a du soleil ; les enfants jouent ; Pierre mange une pomme...), une phrase à deux prédicats est complexe : Pierre parle en mangeant une pomme; les enfants jouent avec une joie évidente; apeuré par les éclairs, le chat s'est réfugié sous l'escalier...). Les phrases à deux prédicats sont complexes parce que le second prédicat est la réduction d'une phrase dans laquelle des éléments ont été effacés. L'effacement ou la réduction ne modifie pas le sens. L'interprétation se fait à travers la reconstruction des éléments effacés.

Le même phénomène de condensation agit aussi au seul niveau du lexique : un verbe décomposable (par ex. chuchoter) est plus complexe qu'un verbe non décomposable (par ex. dire). Le verbe décomposable peut être défini par un verbe élémentaire (et d'autres mots) mais pas l'inverse : chuchoter quelque chose à quelqu'un = dire quelque chose à quelqu'un à voix chuchotée/basse.

Dans les restitutions que nous avons analysées, les prédications complexes, de même que le lexique complexe sont d'autant plus reformulés que les enfants sont grands (Martinot et al, 2009, Martinot, 2010).

Concernant la complexité des procédures de reformulation mises en œuvre, on a constaté un mouvement de complexification (voir tableau 1 ci-dessus) corrélé à l'âge des enfants. La répétition n'est pas en soi une activité complexe même si elle demande une grande attention, de la mémoire et vraisemblablement une bonne compréhension de ce qui a été dit, en revanche transformer deux prédications simples en une prédication complexe, transformer au passif une phrase à l'actif, effectuer une translation, sont des procédures complexes à mettre en œuvre parce qu'elles entraînent plusieurs changements à la fois.

Dans la séquence 11, les prédications 2 et 3 (ci-dessous) concentrent un grand nombre de phénomènes complexes, au niveau syntaxique et sémantique, et parallèlement, les procédures de reformulation se complexifient en fonction de l'âge des enfants :

Séq. 11. Tout à coup, le tronc s'ouvrit et les enfants furent éblouis par la lumière qui inondait l'intérieur de l'arbre. Ils firent quelques pas et l'arbre se referma derrière eux. ER (Pauline, 4) et l'arbre s'ouvra devant les enfants après il y avait de la lumière ER (Camille, 4) et ils ont vu de la lumière XXX

ER (Clara, 4) et après tout d'un coup le tronc de l'arbre se rouvrit et puis il fut ébloui par la lumière

ER (Sacha, 6) et l'arbre s'ouvrit et après ++ ils ils ils étaient éblouis par la lumière et il faisa deux pas et l'arbre se referma derrière eux

ER (Chloé, 6) et puis l'arbre il s'ouvre et puis ils sont éblouis parce que il y a parce qu'il y a la lumière

ER (Antonin, 8) et à un moment s'ouvrit et et et ils étaient éblouis par tant de lumière ER (Marc, 10) puis quelques secondes après il y a eu euh hmm il y a eu une lumière éblouissante puis euh puis ils ont reculé puis l'arbre il s'est refermé

ER (Océanie, 10) au bout de quelques minutes l'arbre s'ouvre et ils voient une mer éblouissante

ER (Paul, 10) et s'ouvrit les enfants éblouis par la lumière qui sortait du tronc ils firent quelques pas ils entrèrent

ER (Léo, 10) et ouvrit une grande porte de euh qui à l'intérieur c'est inondé de lumière il entrait dans l'arbre 
69

Aucun enfant de 4, 6 et 8 ans n'a reformulé les prédications 2 et 3. La prédication 2 seule a été simplifiée par deux enfants de 4 ans (Pauline et Camille), l'une a tenté de répéter (Clara). A 6 ans, les enfants sont un peu plus nombreux que les 4 ans à reprendre la forme passive de la prédication 2 (Sacha, Chloé), et un enfant reformule la relation agentive ( éblouis par la lumière) en une relation de causalité (Chloé), ce qui maintient le même niveau de complexité entre l'ES et l'ER. A 8 ans, quelques enfants (Antonin) tentent de reformuler la grande quantité de lumière évoquée par le verbe métaphorique (inondait).

70 A 10 ans, un enfant reformule les deux prédications (Paul), un autre reformule la prédication 3 en la restructurant et en maintenant la métaphore (Léo), une autre enfant tente de reprendre la métaphore mais en modifie le sens (Océanie) et deux enfants (Marc et Océanie) transforment le verbe conjugué (furent éblouis) en un adjectif (éblouissante), ce qui correspond à une translation (paraphrase formelle).

71 Les reformulations de cette séquence montrent bien l'évolution des reformulations entre 4 et 10 ans : simplification, répétition (4 et 6 ans), une tentative de complexification à 6 ans (Chloé), quelques tentatives de prendre en compte dans la reformulation de la prédication 2 l'information "grande quantité de lumière» de la prédication 3 (8 ans), quelques reformulations sont complexes à 10 ans : transformations et restructurations, un enfant maintient le même degré de complexité structurale en reformulant la relative, et deux enfants maintiennent le degré de complexité sémantique (métaphore). D'autres exemples de reformulation par simplification puis complexification peuvent être consultés dans Martinot (2010).

\section{Quelques suggestions pour utiliser les différentes procédures de reformulation dans le contexte pédagogique}

72 De nombreuses recherches ont analysé les types de reformulations qui sont en lien avec un certain nombre de fonctions pédagogiques de la parole enseignante dont la confirmation, l'explication ou la correction (Garcia-Debanc, 2007, Volteau \& GarciaDebanc, 2008, articles de cette livraison). Cependant, la reformulation produite par les élèves en tant que moyen d'appropriation de la langue en milieu scolaire est peu documentée. Pégaz Paquet (2011) ouvre la voie en décrivant comment les reformulations orales des élèves constituent des outils transférables à la production d'écrit. Nous suggérons qu'en produisant certains types de reformulation, notamment les reformulations paraphrastiques, mais aussi celles qui modifient le sens, l'élève apprend quelque chose sur le fonctionnement de sa langue. En-effet, dans la mesure où la reformulation, telle que nous l'avons définie, permet à l'enfant d'acquérir sa langue maternelle en transformant les énoncés sources conformément aux règles de fonctionnement de la langue cible ${ }^{18}$, nous pouvons exploiter cette pratique spontanée dans le cadre scolaire.

73 L'objectif général des activités de reformulation est de développer la conscience épi- puis métalinguistique des élèves, c'est-à-dire la conscience de ce qui dans la langue est responsable du sens d'un énoncé : choix et ordre des mots, et mode de construction de la prédication. Les élèves doivent repérer, comprendre et expérimenter que l'enjeu d'un énoncé résulte toujours, en premier lieu, de l'articulation du lexique et de la construction dans laquelle est actualisé ce lexique. Ce principe général du fonctionnement de toute

Corela, HS-18 | 2015 
langue peut être exploité en classe à condition de sélectionner les postures reformulatoires caractéristiques d'une tranche d'âge donnée. Comme nous l'avons signalé dans la partie (4), ci-dessus, les reformulations répétitives sont les plus nombreuses (relativement aux autres postures reformulatoires) jusqu'à 6 ans (Martinot, 2003). Cela signifie que les enfants sont attentifs à la forme globale d'un énoncé (prédicatif) qu'ils vont tenter de répéter. L'activité reformulatoire menée en classe peut alors s'appuyer sur cette compétence langagière pour que les élèves produisent non pas de simples reformulations répétitives mais des reformulations qui maintiennent la même construction tout en changeant le sens de l'énoncé source en remplaçant un mot par un autre. On verra ci-dessous comment ce type d'exercice peut avoir un sens pour les élèves (5.1).

74 A partir de 8 ans, la compétence paraphrastique est ou devrait être attestée chez la plupart des enfants. On sait, depuis les travaux de Bernstein $(1958,1975)$ qu'une des causes majeures de l'échec scolaire vient du fait que les enfants issus des classes sociales défavorisées ne disposent que d'un code restreint qui ne leur permet pas de s'adapter à toutes les situations d'énonciation, c'est-à-dire qui ne leur permet pas de donner la même information sous des formes variables, autrement dit de paraphraser un énoncé antérieur, disponible dans leur mémoire. Il s'agirait donc de faire pratiquer très régulièrement, dès le $\mathrm{CE} 1$, des reformulations qui maintiennent le sens et modifient la forme (5.2).

Enfin, l'activité reformulatoire en général permet à l'enfant de poursuivre l'acquisition de sa langue maternelle jusqu'à ce que ses énoncés atteignent le même degré de complexité lexicale et syntaxique que les énoncés produits par des adultes ayant validé une formation secondaire ou ayant été scolarisés jusqu'à 18-19 ans ${ }^{19}$. Au cycle 3 , les exercices de reformulation peuvent être mis au service de la complexification des énoncés, dont l'une des manifestations peut être de produire un plus grand nombre d'informations avec un moins grand nombre de mots. Cet entraînement à la synthétisation est évidemment nécessaire à l'amélioration de la production écrite qui doit se rapprocher peu à peu des attentes académiques (5.3).

76 Ces manipulations permettent de donner davantage de sens aux activités réflexives sur la langue. Toute production écrite ou orale est d'abord une production de sens qui doit être expliquée, la dénomination ou l'étiquetage des catégories passant alors au second plan.

\subsection{Reformuler en gardant la même construction}

Imaginons que l'enseignant propose à ses élèves (moyenne, grande section de maternelle, cours préparatoire, éventuellement cours élémentaire première année) de fabriquer ensemble un petit manuel de langue française pour les enfants non francophones. Ces enfants (en fait tous les enfants) doivent en-effet savoir quels mots vont ensemble et quels mots ne peuvent pas aller ensemble dans le cadre de la construction d'une prédication simple.

A partir d'une telle prédication, par exemple, l'une des phrases du texte Tom et Julie :

La maîtresse tenait par la main une petite fille

les élèves proposent d'autres prédications qui, à la fois, suivent le même modèle structurel que celui de la phrase source (dét $N_{0}\left[V\right.$ par dét $\left.N_{2}\right]$ dét (adj) $N_{1}$ ) et contiennent un 
nouveau mot de façon à ce que le sens de l'énoncé reformulé soit différent du sens de l'énoncé de départ :
a. La fille tenait par la main une petite fille ${ }^{20}$
b. La fée tenait par la main une petite fille
c. Le maître tenait par la main une petite fille
d. *La maitresse tenait par (la poignée + le manche) une petite fille ${ }^{21}$
e. La maîtresse tenait par la main une petite ???

Le travail de recherche lexicale fait sur l'axe paradigmatique permet de constater que le choix de tel nom sujet $\left(\mathrm{N}_{0}\right)$ et de tel verbe (tenir par la main) réduit fortement le choix du nom complément direct $\left(\mathrm{N}_{1}\right)$ (cf. d-e). On peut également remarquer que les noms sujet ( $\mathrm{N}$ $\left.{ }_{0}\right)$ et complément direct $\left(\mathrm{N}_{1}\right)$ des phrases (a-e) sont tous des noms humains, que le complément introduit par la préposition par désigne une partie du corps et que donc dans une construction donnée, on ne peut pas employer indifféremment n'importe quel nom (comparer avec : la maîtresse tenait par la poignée une petite valise). A l'école élémentaire, les catégories grammaticales seront nommées au cours de l'activité.

\subsection{Reformuler en gardant le même sens}

81 A partir du CE1 (élèves de 8 ans), l'exercice inverse du précédent peut être fait. Il s'agit alors de garder le même sens tout en changeant un ou deux éléments de la phrase de départ. Les élèves doivent donc produire des paraphrases. L'entraînement à la maîtrise paraphrastique augmente le stock de paraphrases de chaque élève. La maitrise paraphrastique permet en effet au locuteur ou au scripteur d'ajuster au plus près ce qu'il dit à ce qu'il veut dire, et pour cela, plusieurs énoncés auto-reformulés sont toujours nécessaires, même chez un locuteur/scripteur expert. Tant que l'intention de dire n'a pas trouvé la forme linguistique la plus appropriée, cette intention reste insaisissable. Une première formulation permet au locuteur de révéler ce qu'il veut dire, puis d'évaluer si cette première forme linguistique exprime ce qu'il voulait dire, dans le cas contraire, une deuxième forme linguistique suggère une autre alternative et ainsi de suite ${ }^{22}$. Cette activité doit avoir lieu à l'oral d'abord, l'enseignant écrivant chaque suggestion au tableau ${ }^{23}$. Pour que cette activité prenne tout son sens, on a intérêt à la faire porter sur les trois premières phrases ${ }^{24}$ d'une histoire par exemple. Le changement de forme dans la première phrase entrainant probablement un changement dans les deux suivantes.

Ainsi, si l'on reprend le début de l'histoire de Tom et Julie :

Ce matin-là, la maîtresse est arrivée dans la cour de l'école plus tard que d'habitude.

Elle tenait par la main une petite fille que personne n'avait encore jamais vue.

on voit que parmi les paraphrases possibles (f-l) de la prédication 3 (Elle tenait par la main une petite fille) précédée et suivie du même co-texte, seuls les énoncés (h à $\mathrm{k}$ ) sont possibles dans ce contexte :

f. La maîtresse tenait par la main une petite fille

g. Elle la tenait par la main

h. Elle tenait la main $(\mathrm{de}+\mathrm{a})$ une petite fille

i. Elle tenait une petite fille par la main

j. Elle tenait une petite fille à la main

$\mathrm{k}$. Elle tenait par la main une (fillette + fille)

1. Une petite fille était tenue à la main par la maîtresse

D'une façon plus générale, les activités de reformulation paraphrastique entraînent les élèves à vérifier et à justifier que le sens est inchangé, à décrire les changements formels 
(1 ou 2 par énoncé). Ces activités réflexives permettent aussi de faire employer, de façon active, les termes métalinguistiques dans une véritable activité d'observation. complexification. Or, la difficulté majeure des élèves, y compris dans le secondaire, n'est pas tant d'utiliser une subordonnée à la suite d'une principale mais de construire une phrase à un verbe conjugué contenant plusieurs prédicats, comme par exemple «il marchait en boitant ", " la maîtresse est arrivée à l'école en retard». Autrement dit, les élèves doivent apprendre à condenser dans l'espace d'une phrase (de la majuscule au point final) plusieurs informations d'ordre temporel, spatial, modal. Ils doivent en particulier utiliser des compléments non prévus par la rection verbale et enfin construire des groupes nominaux complexes, de type $N$ prép $N$. Par exemple, un enfant de 6 ans dirait : mon frère a crié et après ma maman a eu très peur, en employant deux prédications simples coordonnées. Un locuteur beaucoup plus avancé dans l'acquisition de sa langue maternelle (12 ans au-moins) dirait : les cris de mon frère ont affolé Maman en employant un nom prédicatif, les cris, puis un verbe causatif complexe ont affolé, décomposable en ont fait que Maman est devenue folle de peur.

90 Le travail sur la complexification de la langue doit résulter d'un premier travail sur la simplification dans lequel un texte d'écrivain, par exemple, sera l'objet d'une réécriture simplificatrice accompagnée de la réflexion sur l'effet produit, sur le caractère moins agréable, esthétique du segment simplifié etc...

91 Le principe méthodologique est de toujours associer la réflexion grammaticale à la réflexion sémantique et pourquoi pas stylistique et esthétique : non seulement, ce 
principe correspond au fonctionnement de la langue mais il permet de développer chez les élèves la sensibilité à ce qui est «bien dit ", de donner du sens aux exercices sur la langue en permettant d'enrichir le lexique des élèves, de manipuler des phrases sémantiquement équivalentes, d'associer les contraintes morphologiques aux contraintes syntaxiques.

Enfin, tous ces exercices peuvent se faire à l'oral, de façon régulière et être réutilisés à l'écrit.

\section{Conclusion}

93 A partir de la définition proposée dans Martinot (1994) de la notion de reformulation, nous avons tenté de montrer premièrement que cette notion est opératoire pour rendre compte de la pratique orale et spontanée de tous les locuteurs, deuxièmement que l'on trouve cette pratique chez les enfants au cours de l'acquisition de leur langue maternelle et troisièmement que cette pratique a valeur explicative du processus acquisitionnel. Les procédures de reformulation expliquent en effet comment l'enfant passe des énoncés adultes qu'il repère aux énoncés qu'il produit, d'abord par des reformulations simplificatrices puis par des reformulations complexifiantes. Les recherches acquisitionnelles doivent en effet rendre compte non seulement des premières acquisitions mais également de la suite du processus qui implique nécessairement le passage d'une langue simple à une langue complexe, achevée. C'est dans ce dernier passage que l'institution scolaire a un rôle particulièrement important à jouer de façon à ce que tous les élèves maitrisent en fin de primaire et dans tous les cas à la fin du premier cycle secondaire, vers 14-15 ans, le fonctionnement grammatical de leur langue maternelle ou de leur langue de scolarisation.

\section{BIBLIOGRAPHIE}

AL AWBALI N., (2011) Acquisition des relatives à antécédent en arabe yéménite, Thèse de doctorat, dir. C. Martinot, Université Paris Descartes.

BERNSTEIN B., (1958), « Some sociological determinants of perception : an inquiry into subcultural differences », British Journal of sociology (Londres), vol. 9, nº1, p. 159-174.

BERNSTEIN B., (1975), Langages et Classes Sociales, Paris, Les Editions de Minuit.

DE BOYSSON-BARDIES B. (1996), Comment la parole vient aux enfants? Paris, Editions Odile Jacob.

FUCHS C., (1978), « Paraphrase et énonciation », Stratégies discursives, p. 257-266.

FUCHS C., (1980), Paraphrase et théories du langage ; contribution à une histoire des théories linguistiques contemporaines et à la construction d'une théorie énonciative de la paraphrase, Thèse de doctorat d'Etat, Université Paris VII.

FUCHS C., (1982), La paraphrase, PUF. 
GARCIA-DEBANC C., (2007), « La reformulation orale : un élément de l'expertise professionnelle », dans Talbot, L. \& Bru, M., Des compétences pour enseigner, Presses universitaires de Rennes, p.151-168.

GÜLICH E. \& KOTSCHI T., (1983), « Les marqueurs de reformulation paraphrastique », Cahiers de linguistique française 5, p.305-351.

GÜLICH E. et KOTSCHI T. (1987), «Les actes de reformulation dans la consultation : la dame de Caluire», Berne, Pierre Bange : p. 16-79.

GEROLIMICH S., MARTINOT C., DE GIOIA M., (2009) « Reformulations définitoires spontanées chez des francophones et des italophones de 6, 8 et 10 ans » in Autour de la définition, 6, éd. Publif@rum, http://www.publifarum.farum.it/ezine_printarticle.php?art_id=148

http://www.publifarum.farum.it/ezine_printarticle.php?art_id=149

HARRIS Z.S, (1969), «The two systems of grammar: report and paraphrase», Transformation and discourse analysis papers 79 [p. 612-92 in papers].

HARRIS Z.S, (1976), Notes du cours de syntaxe, Paris, Le Seuil.

HARRIS Z.S. (1988, 2007) (trad. fr.). Language and Information. New York, Columbia University Press (traduction française: Ibrahim, A.H. \& Martinot, C., La langue et l'information, Paris, CRL.)

IBRAHIM A.H. (2001) « Argumentation interne et enchaînements dans les matrices définitoires ", Langages $\mathrm{n}^{\circ} 142$, p.92-126.

IBRAHIM A.H., (2009), « Les conditions de la prédication dans les langues », dans Ibrahim A.H., Prédicats, prédication et structures prédicatives, Paris, CRL, p. 12-49.

IBRAHIM A.H., (2015), L'analyse matricielle définitoire : un modèle pour la description et la comparaison des langues, Paris, CRL.

IBRAHIM A.H. \& MARTINOT C, (2004), « Les reformulations matricielles lacunaires des enfants », Travaux de Linguistique 48, p.21-40.

LE PESANT D., \& MARTINOT C., (2007) « Pour une approche intégrée de l'enseignement du vocabulaire, de la grammaire et de l'orthographe ", Diptyque 11, Presses universitaires de Namur, p. 123-150.

LEROY-COLLOMBEL M. (2009), «La reformulation dans les interactions adulte-enfant : une analyse longitudinale de $1 ; 06$ à $2 ; 08$ ans ", dans Martinot, C. \& Romero, C., La reformulation: acquisition et diversité des discours, Cahiers de praxématique $\mathrm{n}^{\circ} 52$, p. 59-80.

MARTIN R., (1976), Inférence, antonymie et paraphrase ; éléments pour une théorie sémantique, Paris, Klincksieck.

MARTIN R., (1990), « La définition naturelle », La définition, Paris, Larousse, p. 86-95.

MARTINOT C., (1994), La reformulation dans des productions orales de définitions et explications. (Enfants de maternelle), Thèse de doctorat, dir. Blanche-Noëlle Grunig, Université Paris VIII.

MARTINOT C., (2003), Les acquisitions tardives en français langue maternelle. In Claire Martinot \& Amr H. Ibrahim (éds.), La reformulation : un principe universel d'acquisition (39-69). Paris : Kimé.

MARTINOT C., (2004), Comment les procédures de reformulation organisent-elles les textes ? In Sylvie Porhiel \& Dominique Klingler (éds.), L'unité texte. Pleyben : Perspectives, 215-230.

MARTINOT C., (2005a), Comment parlent les enfants de 6 ans? Pour une linguistique de l'acquisition, Presses Universitaires de Franche-Comté. 
MARTINOT C., (2005b), Qu'est-ce-que la Reformulation? Nécessité d'une redéfinition. » Lublin : Roczniki Humanistyczne (Annals of Arts), vol LIII, fasc. 5, 5-13.

MARTINOT C., GEROLIMICH S., PAPROCKA-PIOTROWSKA U., SOWA M., (2008), « Reformuler pour acquérir sa langue maternelle ? Investigation auprès d'enfants français, italiens et polonais de 6 , 8 et 10 ans ", dans Schuwer, M., Le Bot, M.-C., Richard, E., (dir.), PRAGMATIQUE DE LA REFORMULATION, Presses Universitaires de Rennes, p. 221-239.

MARTINOT C., (2009) « Reformulations paraphrastiques et stades d'acquisition en français langue maternelle ", dans Martinot, C. \& Romero, C., La reformulation: Acquisition et diversité des discours, Cahiers de praxématique ${ }^{\circ} 52$, p.29-57.

MARTINOT C., KUVAC-KRALJEVIC J., BOSNJAK-BOTICA T., CHUR L., (2009), « Prédication principale vs seconde à l'épreuve des faits d'acquisition en allemand, croate et français, langues maternelles ", dans Ibrahim A. H., Prédicats, prédications et structures prédicatives, Paris , CRL, p.50-81.

MARTINOT C., (2010), « Reformulation et acquisition de la complexité linguistique », Travaux de linguistique, $\mathrm{n}^{\circ}$ 61, p.63-96.

MARTINOT C., (2012), « De la reformulation en langue naturelle vers son exploitation pédagogique en langue étrangère : pour une optimisation des stratégies d'apprentissage », Synergies Pologne $\mathrm{n}^{\circ}$ 9, tome 1, p. 63-76.

MARTINOT C., GEROLIMICH S, (2012) Acquisition de la compétence paraphrastique par des enfants francophones et italophones de 4 à 10 ans, in La synonymie au-delà du lexique, éd. Forum, Université de Udine, p.195-228.

MARTINOT C., (2013), « L'acquisition de la causalité est-elle comparable chez tous les enfants ? », Travaux de linguistique $n^{\circ} 66,15-52$.

MARTINOT C., «Construction de l'information dans la langue parlée : repérage de schémas reformulatoires remarquables ", in E. Richard et S. Oriez (éds) : Des organisations dynamiques de la langue orale. Science pour la communication, Peter Lang (sous presse).

MARTINOT C., ROMERO C., (2009) « Présentation : La reformulation : Acquisition et diversité des discours ", dans Martinot, C. \& Romero, C., La reformulation : Acquisition et diversité des discours, Cahiers de praxématique $\mathrm{n}^{\circ} 52, \mathrm{p} .7-18$.

PEGAZ PAQUET A., (2011) Quand l'oral passe à l'écrit : place et rôle de l'oral dans la construction des savoirs linguistiques mis en œuvre à l'écrit, Thèse de doctorat, dir. C. Martinot, Université Paris Descartes.

STRÖBL A., (1977), « Zur Erklärung von funktionalen Paraphrasen », Salzburger Beiträge zur Linguistik 3, Tübingen, Narr, p.373-383.

TUTUNJIU E., (2012) Acquisition des phénomènes linguistiques complexes en roumain langue maternelle (enfants de 6 à 10 ans), Thèse de doctorat, dir. C. Martinot, Université Paris Descartes.

VOLTEAU S., \& GARCIA-DEBANC C., (2008), « Les reformulations de l'enseignant dans quatre situations scolaires : existe-t-il une influence de l'objet enseigné sur les types de reformulation utilisées ? », dans Schuwer, M., Le Bot, M.-C., Richard, E., (dir.), PRAGMATIQUE DE LA REFORMULATION, Presses Universitaires de Rennes, p. 253-268

WARMHOFF S., (1981), « Die Funktion der Paraphrase in Gesprächspsychotherapeutischen Beratungen ", Deutsche Sprache n9, p. 97-118. 
WENZEL A., (1981), « Funktionen Kommunikativer Paraphrasen. Am Beispiel von Gesprächen zwischen Bürgern und Beamten im Sozialamt », dans Schröder P., \& Steger H., (éds.), p. 385-401.

\section{ANNEXES}

Tom et Julie

1) Ce matin-là, la maîtresse est arrivée dans la cour de l'école plus tard que d'habitude. Elle tenait par la main une petite fille que personne n'avait encore jamais vue.

2) Arrivée en classe, la maîtresse a dit : « Les enfants, je vous présente votre nouvelle camarade, elle s'appelle Julie. Tom, la place est libre à côté de toi, Julie sera ta voisine, sois bien gentil avec elle!»

3) Tom était fou de joie à l'idée d'avoir peut être une nouvelle amie. Le soir, chez lui, il a fabriqué une petite boîte ronde, rouge et dorée, pour Julie.

4) Le lendemain matin, dans la cour de l'école, Tom guettait l'arrivée de sa nouvelle petite voisine. Dès qu'il l'a aperçue, il s'est dirigé vers la fillette et lui a tendu la boîte qu'il avait fabriquée pour elle, la veille.

5) Julie aimait tellement cette boîte qu'elle la prenait toujours avec elle. Quand la maîtresse disait : « Sortez vos affaires! », Julie posait délicatement la boîte entre Tom et elle, sur leur table de travail.

6) Un jour, Julie chuchota à Tom : « Ouvre la boîte ! » Tom souleva le couvercle et découvrit un morceau de papier sur lequel Julie avait écrit : « Je t'attends ce soir à $8 \mathrm{~h}$, sous le gros arbre, à l'entrée de la forêt ».

7) Tom avait un peu peur parce qu'il lui était interdit d'aller dans la forêt, surtout la nuit.

8) Mais à $8 \mathrm{~h}$ du soir, il était tout de même au rendez-vous, Julie l'attendait déjà.

9) Sans dire un mot, la petite fille prit la main de Tom et frappa 3 fois sur le tronc du gros arbre.

10) Au bout de quelques minutes, les enfants entendirent un grincement. L'arbre était en train de tourner sur lui-même.

11) Tout à coup, le tronc s'ouvrit et les enfants furent éblouis par la lumière qui inondait l'intérieur de l'arbre. Ils firent quelques pas et l'arbre se referma derrière eux

12) Tom et Julie se trouvaient dans un jardin merveilleux où les fleurs semblaient se parler en chantant. Alors Julie dit à Tom : «Viens, traversons le jardin, il y a une grande fête pour toi, ce soir. Jusqu'à minuit, tu as le droit de demander à notre Roi tout ce que tu veux $»$.

13) Tom a répondu : «Je veux apprendre à parler avec les oiseaux qui savent tout ce qui se passe dans le ciel, avec les poissons qui savent tout ce qui se passe dans l'eau et avec les fourmis qui savent tout ce qui se passe sur la terre ».

14) Et depuis ce jour, Tom est devenu un enfant extrêmement savant. 


\section{NOTES}

1. Pour une présentation claire de l'ensemble des travaux de Harris, voir l'Introduction rédigée par Amr Ibrahim de La langue et l'information (2007 : Trad. française de Harris, 1988).

2. Les exemples (1-5) sont extraits d'autobiographies recueillies sur Radio France Besançon en 1996-97 et analysées dans Martinot (sous presse ).

3. A ce niveau de présentation générale de la reformulation et de la paraphrase, nous nous contentons d'opposer les énoncés sémantiquement équivalents (l'ensemble de tous les types de paraphrases) et les énoncés qui apportent une information différente.

4. Dans le cas des reformulations répétitives, l'invariant est maximal et les modifications minimales : ce peut être l'intonation, le changement de locuteur et dans tous les cas le statut d'énoncé répété.

5. Deux autres procédures de reformulation, que nous aurons l'occasion de décrire plus loin, doivent être ajoutées. La paraphrase explicative, dont l'équivalence ne peut être attestée qu'en discours : (ES) le médecin examine la patiente > (ER) la dame qui est avant moi est encore dans le cabinet. Nous avions désigné, dans nos publications antérieures, la paraphrase explicative par "paraphrase fonctionnelle ou situationnelle ", nous utiliserons dorénavant la désignation de paraphrase explicative qui rend compte des énoncés, divers, dans lesquels le locuteur explique à lui-même ou à son interlocuteur comment il comprend l'énoncé source. La seconde procédure est la paraphrase définitoire, dans laquelle le locuteur fournit la définition analytique d'un mot de l'énoncé source : (ES) l'arbre tournait sur lui-même > (ER) l'arbre faisait des tours sur lui-même 6. Tous les énoncés reformulés ne proviennent pas, loin s'en faut, d'interactions entre l'enfant et l'adulte. Dans le cadre pédagogique d'une classe de grande section de maternelle, on a pu constater (Martinot, 1994) que plusieurs enfants invités à définir, chacun à leur tour, un objet, vont produire une définition qui sera la reformulation de la précédente. Ou encore, que des enfants de Cours préparatoire rassemblés pour raconter, chacun à leur tour, le dernier cauchemar qu'ils ont fait, vont également se reformuler les uns les autres (Martinot, 2005).

7. "L'intuition du moment où, dans l'enchaînement des productions langagières, une forme se parachève au regard d'un contenu est un constituant essentiel de la faculté de langage. C'est aussi la première condition pour reconnaître que dans ce qu'on a entendu ou lu il y a eu une prédication ».

8. Ibrahim (2009: 12) propose une explication extrêmement originale de la reconnaissance de la prédication par les locuteurs d'une langue: «cette intuition de complétude à la fois formelle et sémantique d'unités de tailles très variables qui est indispensable à la production, au découpage et à la reconnaissance des énoncés complexes des discours les plus hautement élaborés comme des discours les plus élémentaires, est un produit dérivé d'une intuition encore plus originelle: celle qui a conduit l'espèce humaine à construire la syllabe selon un schéma harmonique qui a fait coïncider chaque type de syllabe, perçu comme une consonance particulière, avec un type d'accord musical parfait "

9. Projet Acquisition et Reformulation (2003-2010), coordonné par l'auteur de ces lignes, avec la collaboration de Sonia Gerolimich, Urszula Paprocka-Piotrowska, Jelena Kuvac-Kraljevic, Tomislava Bosnjak-Botica, Nour Al Huda Al Awbali (2011) et Elena Tutunjiu (2012).

10. Le texte est lu une seule fois à l'enfant.

11. Al Awbali (2011) a mené cette expérimentation dans plusieurs écoles maternelles de Sana'a, au Yémen, sans aucune difficulté, ni de la part de l'Institution, ni de la part des enfants qui ont produit des reformulations extrêmement créatives (ibid.).

12. Les phénomènes en question sont complexes en français, d'autres phénomènes complexes peuvent être attestés dans les autres langues du projet de recherche.

13. Le signe + note une courte pause. 
14. La définition analytique de voisine dans Julie sera ta voisine est: Julie sera la fillette qui prendra place à côté de toi ou qui s'assoira à côté de toi.

15. Dans la suite de nos travaux et en particulier dans le nouveau projet que nous menons actuellement sur l'acquisition de la complexité linguistique, nous avons été amenées à introduire un nouveau type de reformulation : la paraphrase synthétique. Ex. ES : La maîtresse est arrivée dans la cour de l'école ... elle tenait par la main une petite fille > ER : la maîtresse amenait une petite fille dans la cour de l'école. Le sens des deux verbes se condense en un seul verbe.

16. Comme déjà dit auparavant, les analyses ont porté aussi sur d'autres langues que le français. Les résultats comparatifs obtenus jusqu'à ce jour convergent avec les résultats obtenus auprès des enfants francophones.

17. Exemple de restructuration: Paul a chargé des caisses dans le camion > Paul a chargé le camion de caisses.

18. Un enfant francophone par exemple, dès l'âge de 3 ans, ne reformule jamais un énoncé antérieur en modifiant l'ordre sujet-verbe, alors que cette permutation est possible dans d'autres langues.

19. Nous introduisons ce critère académique à titre de proposition à valider. Il n'y a aucune raison de considérer que tous les locuteurs adultes d'un même idiome produisent des énoncés ayant le même degré de complexité lexicale et syntaxique. Il nous semble que ce que l'on appelle la « langue standard » est proche du parler d'un adulte ayant eu une formation secondaire et que ce parler «moyen» contient la plupart des phénomènes complexes de la langue en nombre suffisant.

20. Introduire aussi l'énoncé suivant: ( $\left(a^{\prime}\right)$ la fille tenait par la main une fille. Demander pourquoi (a) est plus acceptable que (a'). Mais aussi, pourquoi on ne dirait pas : (a") la chaise tenait par la main une fille.

21. L'astérisque note un énoncé agrammatical.

22. En dehors du fait que le passage de la pensée à la parole reste une énigme, aussi bien du point de vue de la production langagière que des processus de compréhension, nous ne reprenons pas à notre compte une expression largement répandue qui parle de "mise en mots " à partir d'une représentation abstraite. Sans nier la base psychique de la plupart des activités humaines dont la production langagière, le cheminement de la pensée et du raisonnement se réalise dans et par la langue, c'est la langue qui nous permet de penser et non la pensée qui nous permet de parler.

23. Nous ne rentrons pas dans le détail de la mise en œuvre pédagogique qui variera selon que les enfants sont en CE2 ou en CM2. En particulier, l'enseignant a le choix entre deux grandes alternatives: soit il propose les paraphrases (ou une partie) aux élèves et ces derniers discutent des possibilités d'insertion dans un texte, soit les élèves doivent trouver les paraphrases, en «oubliant» le texte de départ puis en y revenant ensuite pour discuter des possibilités d'insertion. Ce type d'activité peut se faire en collectif mais aussi sous la forme d'ateliers d'écriture à partir du CE1.

24. Le terme de " phrase » correspond ici à celui de " proposition». La ponctuation nous indique qu'il y a deux phrases; l'analyse grammaticale qu'il y a trois "propositions" et l'analyse syntaxique qu'il y a quatre prédications, la première "phrase " étant constituée de deux prédications simples : La maîtresse est arrivée dans la cour de l'école \& La maîtresse est arrivée plus tard que d'habitude (cf. 3.3. ci-dessus).

25. Ce type d'activité reformulatoire nous semble particulièrement intéressante à exploiter en classe, parce qu'elle entraîne les élèves à prendre en compte tous les aspects sémantiques d'un verbe (ou d'un nom, d'un adjectif, d'un adverbe) comme par exemple ici, le fait que " marcher » ou « boiter » correspondent à un déplacement vers l'avant, alors que l'on peut marcher ou boiter vers l'arrière. Dans ce cas, on est obligé de préciser « marcher à reculons », « boiter à reculons ». 


\section{RÉSUMÉS}

L'auteure rappelle la définition technique qu'elle a donnée en 1994 de la reformulation et montre que cette définition permet de rendre compte à la fois des discours oraux et spontanés des adultes mais aussi des enfants. Cette définition permet également d'expliquer comment les enfants acquièrent leur langue maternelle en transformant les énoncés adultes selon un principe unique. Ces différents types du même principe de reformulation sont corrélés à l'âge des enfants et au degré de complexité des énoncés sources. Ils révèlent parallèlement l'aptitude transformiste de chaque enfant. Cette aptitude à la reformulation peut et doit être systématiquement entraînée dans le cadre scolaire pour accroître le stock paraphrastique de chaque enfant, condition indispensable à la maîtrise de la langue maternelle.

The author reminds the technical definition of reformulation she proposed in 1994 and shows that this definition makes it possible to account both for adults' spontaneous speech productions and for children's productions. This definition also explains how children acquire their first language by transforming adult utterances following a unique principle. These several types of the same principle of reformulation are correlated with the age of children and with the complexity degree of source utterances. At the same time, they show the transformist ability of each child. This ability to reformulate can and has to be systematically trained in the class-room so that the paraphrastic stock of each child grows. This is in fact an essential condition to command the first language.

\section{INDEX}

Keywords : reformulation, constructing a meaning, acquisition of the first language, complexity, types of paraphrases, teaching of the first language.

Mots-clés : reformulation, construction du sens, acquisition de la langue maternelle, complexité, types de paraphrases, didactique de la langue maternelle.

\section{AUTEUR}

\section{CLAIRE MARTINOT}

Université Paris Sorbonne

EA 4509 STIH, Sens Textes Informatique Histoire 